\title{
Corrigendum: Loss of flight promotes beetle diversification
}

Hiroshi Ikeda, Masaaki Nishikawa \& Teiji Sota

Nature Communications 3:648 doi: 10.1038/ncomms1659 (2012); Published 31 Jan 2012; Updated 23 Oct 2012

In Fig. $2 \mathrm{~b}$ of this Article, the distribution map for Necrodes littoralis was inadvertently duplicated under the label Necrophila brunnicollis. The correct version of Fig. 2 appears below.

a

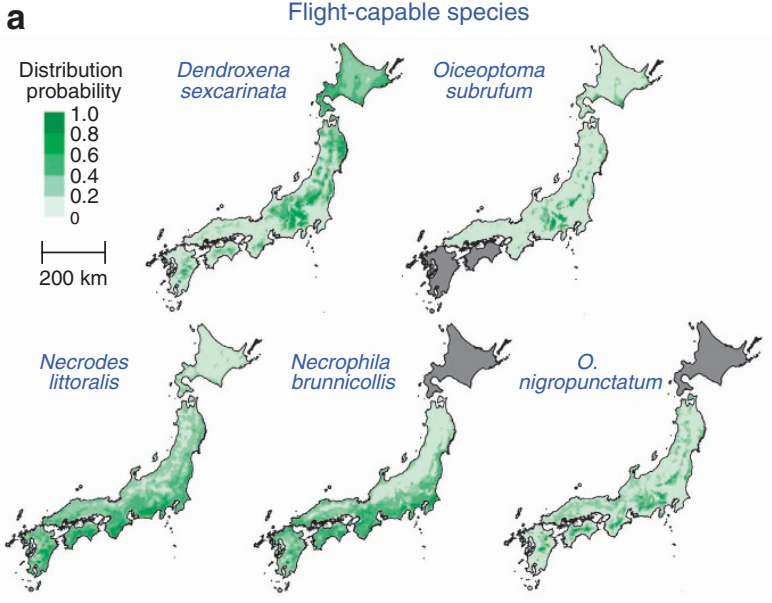

Dimorphic species

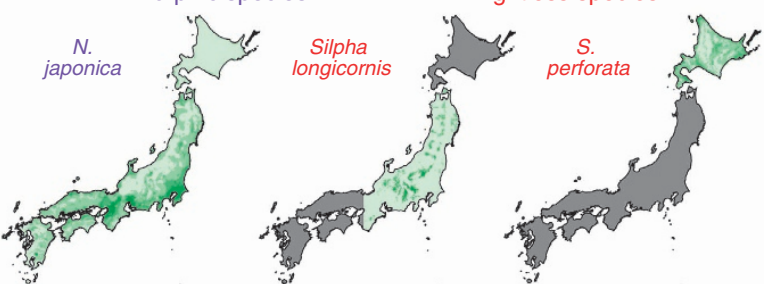

b
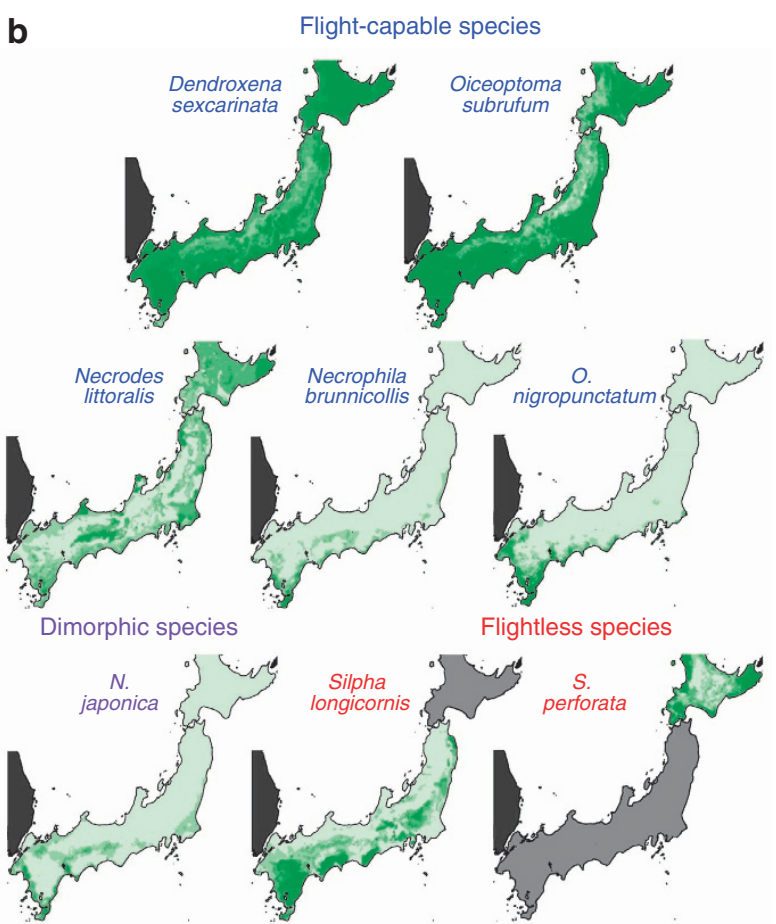